\title{
Inclusive reconstruction of hadron resonances in elementary and heavy-ion collisions with HADES
}

\author{
Georgy Kornakov ${ }^{1, \star}$ \\ for the HADES Collaboration \\ ${ }^{1}$ Technische Universität Darmstadt, Germany
}

\begin{abstract}
The unambiguous identification of hadron modifications in hot and dense QCD matter is one of the important goals in nuclear physics. In the regime of $1-2 \mathrm{GeV}$ kinetic energy per nucleon, HADES has measured rare and penetrating probes in elementary and heavy-ion collisions. The main creation mechanism of mesons is the excitation and decay of baryonic resonances throughout the fireball evolution. The reconstruction of shortlived $(\approx 1 \mathrm{fm} / \mathrm{c})$ resonance states through their decay products is notoriously difficult. We have developed a new iterative algorithm, which builds the best hypothesis of signal and background by distortion of individual particle properties. This allows to extract signals with signal-to-background ratios of $<1 \%$.
\end{abstract}

\section{Introduction}

The mechanism of mass generation of hadrons stays an open question. Although the Higgs mechanism successfully explains mass generation of the three families of fermions, leptons and quarks, and the electroweak gauge bosons $\mathrm{W}^{ \pm}$and $\mathrm{Z}$, the way how matter, i.e. neutrons and protons, obtains its mass remains unclear. One of the scenario relates the QCD chiral condensate $\langle\bar{q} q\rangle$ to the mass. In vacuum, the chiral symmetry is spontaneously broken [1]. In AA collisions, depending on the energy and system size, chiral symmetry can be partially restored. These changes are reflected in the modification of the spectral functions of hadrons. It was shown that from relativistic (SIS18, SPS, AGS) to ultra-relativistic collisions (RHIC, LHC) baryons and anti-baryons play an important role in the modification of the spectral functions of the $\rho, \omega$ and $\phi$ mesons [2]. One can observe these modifications best in the di-lepton decay channel, although it might be that reconstruction of $\pi^{+} \pi^{-}$decays could provide valuable information as well [3].

For this reason, the HADES (High Acceptance Di-Electron Spectrometer) [4] makes systematic measurements of elementary and heavy-ion collisions $\left(p N, p A, \pi^{-} N, \pi^{-} A\right)$ at intermediate temperatures and high baryon chemical potential. HADES is located at the SIS-18 accelerator at GSI, Darmstadt, Germany. Accelerated ion beams, as well as protons and a secondary pion beam are available to be collided with a fixed target consisting of a liquid hydrogen, polyethylene, carbon, Au or W. The detector has low mass tracking detectors and a superconducting toroidal magnet, both divided in equal sextants, providing a momentum resolution better than $2 \%$ for $1 \mathrm{GeV} / \mathrm{c}$ protons and pions. Hadron and lepton reconstruction and identification is based on the measurement of the specific energy loss

^e-mail: g.kornakov@gsi.de 


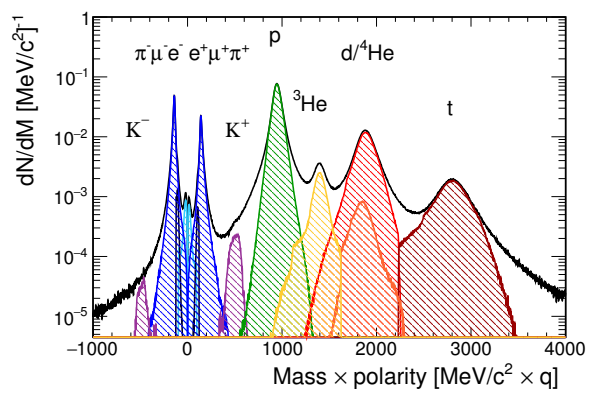

Figure 1. Reconstructed mass from momentum and velocity in HADES after particle identification in $\mathrm{Au}+\mathrm{Au}$ collisions at $1.23 \mathrm{GeV} / \mathrm{u}$.

and the time of flight technique. An example of the mass distribution from combined time of flight and momentum measurements is shown in Fig. 1.

\section{Method, results and conclusions}

The reconstruction of short-lived resonances by means of measurement of the four-momentum of their decay products into hadrons is obfuscated by random combinations, called combinatorial background. In order to overcome the limitations methods commonly used for that purpose, as the different event-mixing techniques [5], we have developed an iterative algorithm that prevents shape distortions and need of weakly-defined normalization factors [6]. The method uses random rotations of particles within the same event, allowing to reduce statistical fluctuations by increasing the number of rotations of one particle. The combinatorial background hypothesis is improved in every iteration step. Correlations appearing from a limited acceptance, occupancy and efficiency are taken into account as well as correlations due to the event reaction plane.

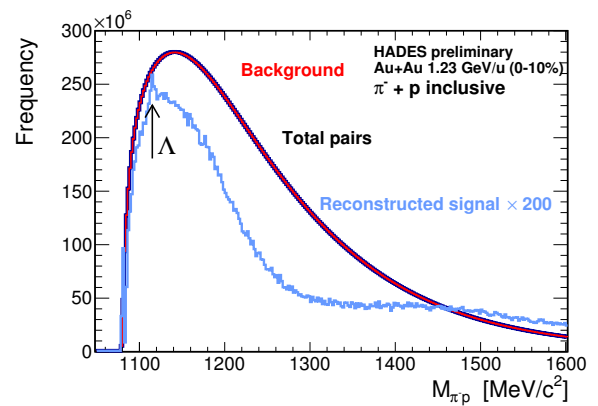

Figure 2. Reconstructed inclusive $\pi^{-} p$ signal measured by HADES in the $10 \%$ most central Au+Au collisions at 1.23 $\mathrm{GeV} / \mathrm{u}$. The total measured signal is shown with a black line together with the obtained combinatorial background after applying the iterative technique. Their difference is represented in blue after scaling by a factor of 200 for visual comparison with the total measured distribution. The method performs efficiently in environments with signal-to-background rations smaller than $1 \%$.

An example of a reconstructed inclusive signal of $\pi^{-} p$ pairs from the $10 \%$ most central $\mathrm{Au}+\mathrm{Au}$ at $1.23 \mathrm{GeV} / \mathrm{u}$ collisions is shown in Fig. 2. The narrow $\Lambda$ peak followed by a broad continuum that can be attributed to resonance decays, e.g. $\Delta, N^{*}$, are identifiable. As the transformations used preserve energy and momentum, the iterative method works efficiently for elementary reactions as well. In this case, the algorithm behaves similarly to random phase-space generators [7], but includes the detector response obtained from real data, that is to say acceptance, efficiency and granularity. The technique was successfully tested with data from $\pi^{-}$induced reactions on a carbon target at an incident momentum of $1.7 \mathrm{GeV} / \mathrm{c}$ measured by HADES. The reconstructed signal in the invariant-mass - pair transverse momentum plane of $\pi^{+} \pi^{-}$and $\pi^{-} p$ are shown in Fig. 3.

In conclusion, the developed method for calculating the combinatorial background is suitable for multi-differential analysis of heavy-ion collisions as well as elementary reactions. The method was 
tested with real data measured by HADES. The reconstruction of the inclusive production of resonances in the $\pi^{ \pm} p$ and $\pi^{+} \pi^{-}$channels in both $\mathrm{Au}+\mathrm{Au}$ and elementary reactions will help to understand better the production mechanisms, medium and nuclear effects and also sets constraints on some hadron sources of the di-lepton cocktail.
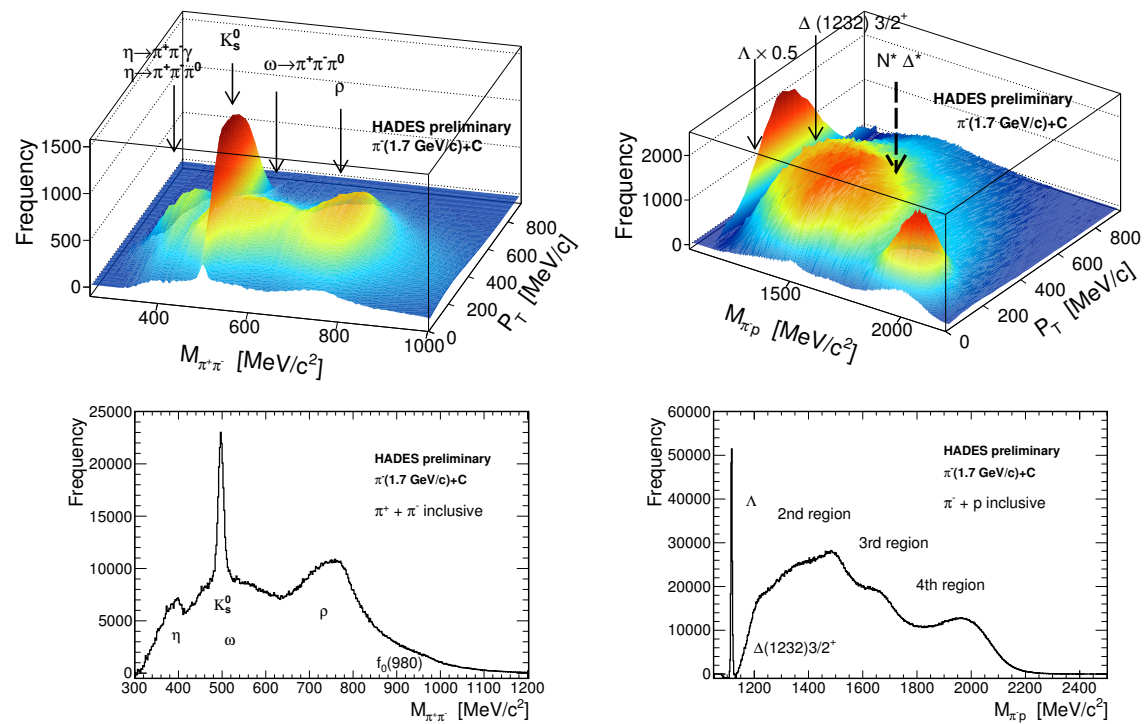

Figure 3. Inclusive $\pi^{+} \pi^{-}$(left) and $\pi^{-} p$ (right) raw spectra measured by HADES in pion induced reactions on a carbon target at an incident momentum of $1.7 \mathrm{GeV} / \mathrm{c}$. Upper panels: invariant mass - pair $P_{T}$ distributions. Lower panels: one-dimensional projections of the invariant masses.

The HADES Collaboration gratefully acknowledges the support by grants PTDC/FIS/113339/2009 LIP Coimbra; NCN 2013/10/M/ST2/00042 SIP JUC Cracow; 2013 /10/M/ST2/00042 Helmholtz Alliance HA216/EMMI GSI Darmstadt; VH-NG-823, Helmholtz Alliance HA216/EMMI TU Darmstadt; 05P12CRGHE HZDR Dresden; Helmholtz Alliance HA216/EMMI, HIC for FAIR (LOEWE), GSI F\&E Goethe-University Frankfurt; MLL, DFG ECLUST 153, VH-NG-330, BMBF 06MT7180, GSI TMLRG1316F, BMBF 05P15WOFCA, TU Munchen; BMBF:05P12RGGHM JLU Giessen; UCY/3411-2311 University Cyprus; CNRS/IN2P3 IPN Orsay; MSMT LG 12007, AS CR M100481202, GACR 13-06759S NPI AS CR, Rez and EU Contract No. HP3283286.

\section{References}

[1] S. Weinberg, Phys. Rev. Lett. 18, 507 (1967)

[2] R. Rapp, H. van Hees, Eur. Phys. J. A 52, 257 (2016)

[3] R. Rapp, Nucl. Phys. A 725, 254 (2003)

[4] G. Agakishiev et al., [HADES Collaboration], Eur. Phys. J. A 41, 243 (2009)

[5] D. L. Hôte, Nucl. Instr. Meth. Phys. Res. A 337, 544 (1994)

[6] G. Kornakov, Manuscript in preparation

[7] F. James, Monte Carlo Phase Space, CERN 68-15, (1968) 